\section{Fluidic lens laparoscopic zoom camera for minimally invasive surgery}

\author{
Frank S. Tsai, ${ }^{\mathrm{a}, *}$ Daniel Johnson, ${ }^{\mathrm{a}}$ Cameron S. Francis, \\ Sung Hwan Cho, ${ }^{a}$ Wen Qiao, ${ }^{a}$ Ashkan Arianpour, ${ }^{a}$ \\ Yoav Mintz, ${ }^{\text {c }}$ Santiago Horgan, ${ }^{\text {b Mark Talamini, }}$, and \\ Yu-Hwa Lo ${ }^{a}$ \\ aUniversity of California, San Diego, Department of \\ Electrical and Computer Engineering, 9500 Gilman Drive, \\ Mail Code 0405, La Jolla, California 92093-0407 \\ bUniversity of California, San Diego, Department of Surgery, \\ School of Medicine, 9500 Gilman Drive, Mail Code \\ 0602, La Jolla, California 92093-0407 \\ ${ }^{\mathrm{c}}$ Hadassah-Hebrew University Medical Center, Department \\ of General Surgery, Jerusalem, 91120, Israel
}

\begin{abstract}
This work reports a miniaturized laparoscopic zoom camera that can significantly improve vision for minimally invasive surgery (MIS), also known as laparoscopic surgery. The laparoscopic zoom camera contains bioinspired fluidic lenses that can change curvature and focal length in a manner similar to the crystalline lenses in human eyes. The traditional laparoscope is long, rigid, and made of fixed glass lenses with a fixed field of view. The constricted vision of a laparoscope is often an inconvenience and plays a role in many surgical injuries. To further advance MIS technology, we developed a new type of laparoscopic camera that has a total length of less than $17 \mathrm{~mm}$, greater than $4 \times$ optical zoom, and 100 times higher sensitivity than today's laparoscope allowing it to work under illumination as low as 300 lux. All these unique features are enabled by the technology of bioinspired fluidic lenses having a dynamic range over $100 \mathrm{di}-$ opters and being convertible between a convex and concave shape. () 2010 Society of Photo-Optical Instrumentation Engineers. [DOI: 10.1117/1.3420192]
\end{abstract}

Keywords: laparoscopic camera; fluidic lens; zoom; minimally invasive surgery.

Paper 09572LR received Dec. 24, 2009; revised manuscript received Mar. 19, 2010; accepted for publication Apr. 1, 2010; published online May 24, 2010.

\section{Introduction}

Classically, surgical access to the abdominal cavity has been accomplished by large incisions through the abdominal wall. Such "open" procedures allow direct visualization and manipulation of the abdominal organs, but produce considerable postoperative pain and significant scars. In contrast, minimally invasive surgery (MIS) requires much smaller incisions $(\sim 1 \mathrm{~cm}$ or less $),{ }^{1}$ resulting in less pain, ${ }^{2}$ faster recovery times, ${ }^{3}$ fewer wound infections, ${ }^{4}$ and minimal scarring. The critical technology enabling MIS is the video laparoscope, a long (30 to $45 \mathrm{~cm}$ ), thin (5 to $15 \mathrm{~mm}$ diam) rigid cylinder

*Address all correspondence to: Frank S. Tsai, University of California, San Diego, Department of Electrical and Computer Engineering, 9500 Gilman Drive, Mail Code 0405, La Jolla, CA 92093-0407. Tel: 858-334-5001; Fax: 858-5588378; E-mail: frank.sftsai@gmail.com containing rod lenses, coupled proximally to a video camera. It acquires real-time images from inside the abdomen for display on operating room monitors, obviating the need for large incisions to visualize abdominal contents.

A typical laparoscopic surgery is performed using three to five $1 \sim 2 \mathrm{~cm}$ "keyhole" incisions through which plastic sheaths called trocars are inserted, creating transabdominal ports for the laparoscope and surgical instruments, as shown in Figs. 1(a) and 1(b). ${ }^{5,6}$ The abdomen is then insufflated with $\mathrm{CO}_{2}$ gas to create a working space, as seen in Fig. 1(b).

Recent innovations in MIS minimize the total number of skin incisions required during a procedure. Cholecystectomy and appendectomy have been performed using just a single incision, through which the laparoscope and surgical instruments simultaneously access the abdominal cavity. ${ }^{7,8}$ Another important advancement in MIS is natural orifice surgery, in which access to the abdomen cavity occurs through the wall of either the stomach (via the mouth), the vagina, or the colon (via the anus). ${ }^{9}$ This potentially eliminates the need for any external incisions. Challenges faced during single incision and natural orifice surgery demonstrate the limitations of current laparoscopes. In both types of surgery, the laparoscope and instruments share a single, crowded access site. ${ }^{10}$ The limited space results in physical and visual interference between the laparoscope and instruments inside the body, and physical interference between camera operator and surgeon outside the patient body. Also, since laparoscopes lack autofocusing capability, they are designed to have very long depth of focus, and strong illumination $(\sim 20,000 \mathrm{lux})$ is required. The only way to provide such a strong illumination is from an external light source delivered to the laparoscope through a bulky optical bundle.

To improve the feasibility of cutting-edge MIS procedures, we propose that the ideal laparoscopic camera is one that can be mounted onto the internal surface of the abdominal wall, has optical zoom and autofocus capability, and does not require strong external lighting, as shown in Fig. 2. The camera has to be small enough to pass through a small incision into the abdomen. Zoom capability is essential, since surgeons need the ability to see wide views of organs to provide spatial orientation and anatomical context, as well as close-up views when operating on the tiny substructures of organs. To eliminate external lighting, the optical system should be fast enough (i.e., small enough f-number) so that it can operate under internal light emitting diode (LED) lighting. The $\mathrm{CO}_{2}$ filled abdominal cavity has roughly $15 \mathrm{~cm}$ of working space, thus the optical system has to be short and have shortest working distances less than $10 \mathrm{~cm}$. Such an advanced laparoscopic camera is difficult to achieve with traditional lenses, prompting us to pursue unconventional lens technology (i.e., a bioinspired fluidic lens) to meet these challenges.

Conventional lenses are made of rigid materials that impart a fixed curvature. In contrast, the human crystalline lens has a curvature that changes as force or pressure is applied. In vivo, this allows the eye to focus on both near and far objects without inward and outward translocation of the lenses. Inspired by nature, we designed a bioinspired fluidic lens that possesses tunable curvature. ${ }^{11}$ Different approaches have been proposed for making curvature changing lenses, such as di-

1083-3668/2010/15(3)/030504/3/\$25.00 @ 2010 SPIE 

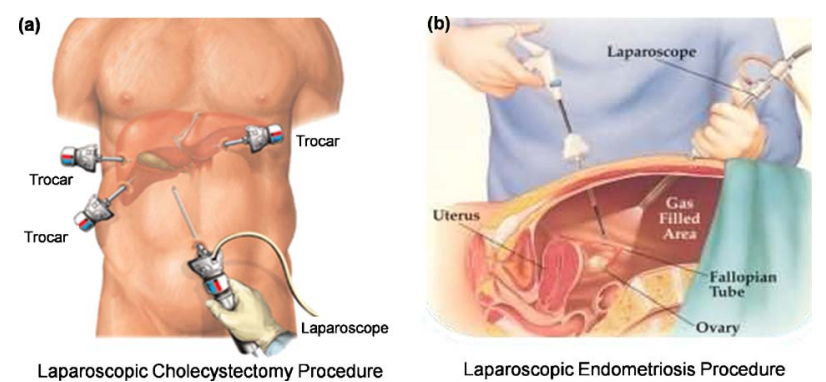

Fig. 1 Examples of laparoscopic surgery.

electrophoretic lens, ${ }^{12,13}$ electrowetting lens, ${ }^{14}$ elastomermembrane fluidic lens, ${ }^{15-17}$ etc. However, to build a high performance zoom camera, the key criteria for choosing the technology of a tunable lens are: 1 . ability to switch between concave and convex lenses; 2. tuning range; 3 . scalability; and 4. mechanical and thermal stability. Compared to all other tunable lens technologies, fluid-filled, elastomer-membrane bioinspired fluidic lenses perform the best in all of these areas. $^{18}$

\section{Design and Fabrication}

Because bioinspired fluidic lenses can easily switch between concave/convex lenses and have an ultrawide tuning range, a zoom lens can be constructed by two fluidic lenses, as shown in Fig. 3. To zoom in, the fluidic lenses are tuned to a telephoto configuration, as shown in Fig. 3(a). To zoom out, the fluidic lenses are tuned to a reverse-telephoto configuration, as shown in Fig. 3(b). Continuous zoom can be achieved by tuning both fluidic lenses to change the effective focal length and to control the back focal length. Such a system was designed and simulated with ZEMAX (Bellevue, Washington), a ray tracing software. Due to the large tuning range of the fluidic lens, over $4 \times$ optical zoom is achieved in a total track length of $17 \mathrm{~mm}$, which is difficult to achieve with conventional zoom lenses made of rigid lenses. Unlike today's laparoscope having an f-number greater than 100, the fluidic zoom lens has a much lower (5 to 10) f-number, thus is 100 times more sensitive to light than conventional laparoscopes. The greatly enhanced sensitivity allows internal LED lighting, eliminating the need for external lighting through a fiber bundle. The main source of aberration in the optical system shown in Fig. 3 is field curvature. The effect of field curvature can be seen in Figs. 4(a) and 4(b), where the edge of the image becomes slightly more blurry.

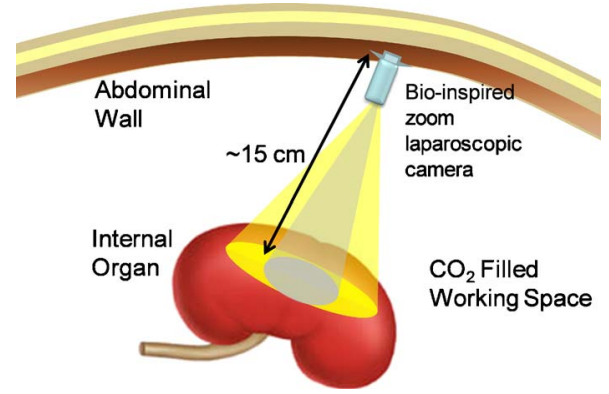

Fig. 2 Conceptual idea for the operation of advanced laparoscopic camera for MIS.
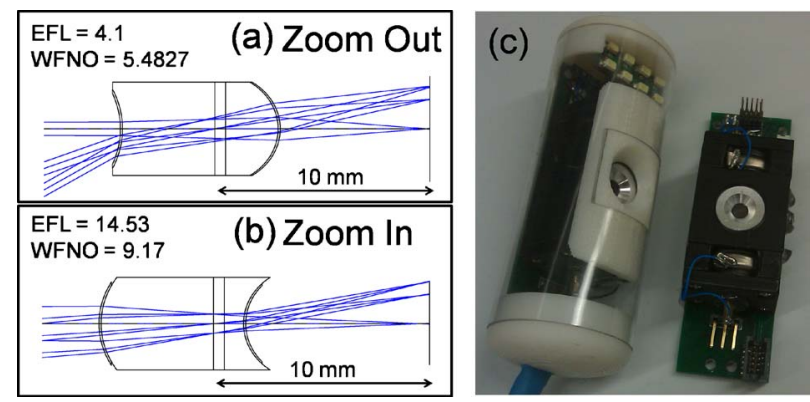

Fig. 3 Ray tracing (ZEMAX) simulation of fluidic zoom lens and picture of fabricated surgical camera. By tuning the curvature of the front and back lenses, the optical system can switch between (a) a reversetelephoto (wide-angle) system to zoom out and (b) a telephoto system to zoom in. (c) The fabricated laparoscopic camera sealed for placement into the abdominal cavity. The camera consists of imaging optics, actuators, electronics, and LED. Pictured right, without an enclosure, is the optics/actuator module attached to the CMOS sensor board.

Based on the design by ZEMAX, the lens module was fabricated and attached onto a 1/3-in. optical format, two megapixel CMOS sensor (OV2630, OmniVision). The system is shown in Fig. 3(c). To control the fluidic lens curvature, an electrochemical cell module that functions as a membrane gas pump is integrated into the camera system. The pump has no moving parts and is electrically controlled. The current design has a total volume of around $0.5 \mathrm{~cm}^{3}$ for the main body of the camera and a response time of $5 \mathrm{sec}$ to switch from extreme zoom in to extreme zoom out, both of which can be further reduced with process optimization. Limited by space, the details of the actuator and its integration with the fluidic lens will be discussed in a separate paper.

\section{Experimental Results and Conclusion}

Following the successful fabrication of the surgical camera, it was tested on a live pig in an operating room. The pig was placed under general anesthesia with its abdomen incised, and the camera was inserted into the abdominal cavity. Figure 4 shows representative images in zoom-in and zoom-out modes at a lighting level of 1500 lux. High-definition (HD), high quality images were obtained over the entire zoom range at a lighting level as low as 300 lux. When zoomed out, the entire surgical field was within the field of view and the whole organ can be seen. When zoomed in, the details of blood vessels were visible to facilitate precise surgical operation. In com-
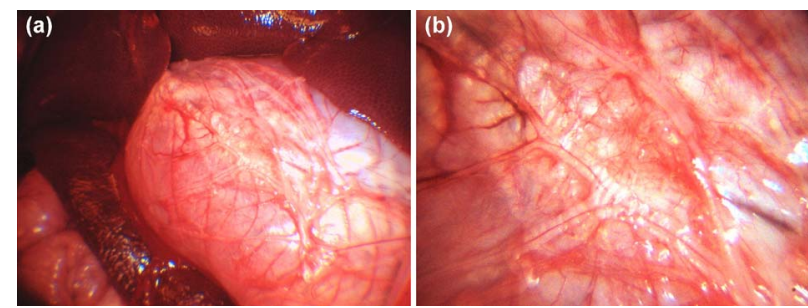

Fig. 4 Images of the organ of a live pig in the operation room taken from our camera. (a) is the zoomed-out view, and the entire stomach can be seen. (b) is the zoomed-in view showing the details. The camera was $12 \mathrm{~cm}$ from the organ, and lighting was provided from an integrated white LED. 

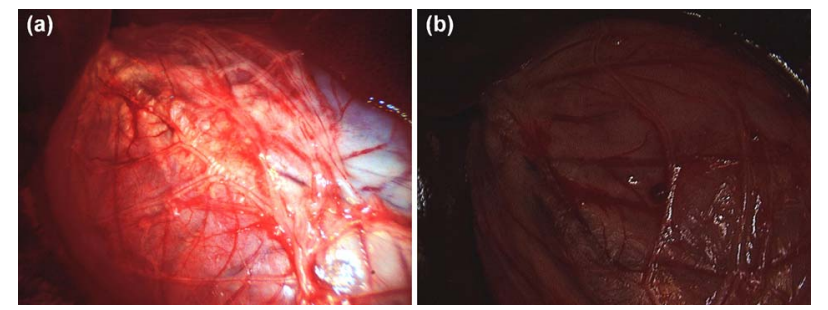

Fig. 5 Comparison of our device with a commercial laparoscope under the same LED lighting. (a) is the image produced by our device and (b) is the image produced by a $10-\mathrm{mm}$ commercial laparoscope under the same LED lighting condition. To match the field of view of (a), the laparoscope was at a distance of $4 \mathrm{~cm}$ from the stomach, while our device was at a distance of $12 \mathrm{~cm}$. The extra distance provides surgeons with extra working space.

parison with commercial laparoscopes, images were also obtained with a Stryker 10-mm laparoscope at the same illumination level used for our camera. Figure 5(a) shows the image taken from our camera, and Fig. 5(b) is the image taken from the state of the art Stryker's laparoscope. For aforementioned reasons (i.e., large f-number), the commercial laparoscope was unable to function under low light conditions. Also, to maintain the same field of view, the distance between the camera and the stomach for a bioinspired fluidic zoom lens was $12 \mathrm{~cm}$, while the laparoscope was only $4 \mathrm{~cm}$. Hence, the bioinspired fluidic zoom provides more working space for surgical tools to operate on the surgical site without physical interference. As a comparison, Fig. 6(a) is taken from our camera with LED lighting, while Fig. 6(b) is taken from a commercial laparoscope with a 300-W fiber-coupled xenon lamp. Both cameras produce similar HD quality images. To analyze the resolution of the medical camera, an ISA/ISO resolution chart (Edmond Optics, Barrington, New Jersey) was placed in front of the surgical camera. Overall, the camera has a resolution better than $600 \mathrm{LW} / \mathrm{PH}$ (line width per picture height) on a 1/3-in. image sensor for all zoom positions.

MIS is a rapidly progressing field driven by the promise of less postoperative pain, shorter recovery time, and nearly invisible scars. As new procedures present challenges to current laparoscopic technology, the need for a new type of laparoscopic camera that can be mounted onto the abdominal wall rises. Such a camera should be small, able to produce optical zoom and high image quality, and work in low light conditions without requiring attachment of a thick fiber optic cable. Taking cues from the optics of human eyes, we developed the fluidic lens and applied the wide tuning range of the lens to
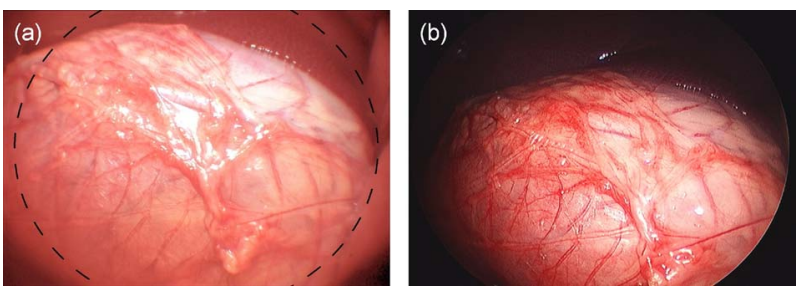

Fig. 6 Comparison of the image quality given sufficient lighting between (a) our device and (b) a commercial laparoscope. The illumination for our camera is 1500 lux, and the illumination for the laparoscope is 20,000 lux provided by an external xenon lamp. demonstrate a laparoscopic zoom camera that is small (17-mm total track, 4-mm clear aperture), has greater than $4 \times$ optical zoom, and works under 300 lux of lighting. With mechanical designs not discussed here, the camera can be inserted through a small incision and be mounted to the inner abdominal wall, offering the image quality and versatility that better approximates open surgery.

\section{Acknowledgments}

The work is supported by a TATRC grant and UC Chancellor's Multidisciplinary Research Initiative. The authors would like to acknowledge the useful technical discussions with Jack Tzeng, Truong Nguyen, UCSD Calit2, and UCSD Department of Surgery. The authors also thank the technical support of the staff of the Nano3 Facility in Calit2.

\section{References}

1. R. McCloy, D. Randall, S. A. Schug, H. Kehlet, C. Simanski, F Bonnet, F. Camu, B. Fischer, G. Joshi, N. Rawal, and E. A. M. Neugebauer, "Is smaller necessarily better? A systematic review comparing the effects of minilaparoscopic and conventional laparoscopic cholecystectomy on patient outcomes," Surg. Endosc 22, 2541-2553 (2008).

2. K. L. Chan, W. C. Hui, and P. K. Tam, "Prospective randomized single-center, single-blind comparison of laparoscopic vs open repair of pediatric inguinal hernia," Surg. Endosc 19, 927-932 (2005).

3. H. S. Tilney, R. E. Lovegrove, S. Purkayastha, A. G. Heriot, A. W. Darzi, and P. P. Tekkis, "Laparoscopic vs open subtotal colectomy for benign and malignant disease," Colorectal Dis. 8, 441-450 (2007).

4. J. E. Varela, S. E. Wilson, and N. T. Nguyen, "Laparoscopic surgery significantly reduces surgical-site infections compared with open surgery," Surg. Endosc 24, 270-276 (2010).

5. See http://www.laparoscopicsurgeryinfo.com/procedure.htm.

6. See http://www.fertilityalabama.com/fertility-tests-laparoscopy.html.

7. E. R. Podolsky, S. J. Rottman, H. Poblete, S. A. King, and P. G. Curcillo, "Single port access (SPA) cholecystectomy: a complete transumbilical approach," J. Laparoendoscopic Adv. Surg. Tech. 19, 219-222 (2009).

8. K. E. Roberts, "True single-port appendectomy: first experience with the puppeteer technique," Surg. Endosc 23, 1825-1830 (2009).

9. S. Horgan, J. P. Cullen, M. A. Talamini, Y. Mintz, A. Ferreres, G. R. Jacobsen, B. Sanndler, J. Bosia, T. Savides, D. W. Easter, M. K. Savu, S. L. Ramamoorthy, E. Whitcomb, S. Agarwal, E. Lukacz, G. Dominguez, and P. Ferraina, "Natural orifice surgery: initial clinical experience," Surg. Endosc 23, 1512-1518 (2009).

10. Y. Mintz, S. Horgan, J. Cullen, D. Syuart, E. Falor, and M. A. Talamini, "NOTES: a review of the technical problems encountered and their solutions," J. Laparoendoscopic Adv. Surg. Tech. 18, 583-587 (2008).

11. F. S. Tsai, S. H. Cho, Y.-H. Lo, B. Vasko, and J. Vasko, "Miniaturized universal imaging device using fluidic lens," Opt. Lett. 33, 291-293 (2008).

12. C. C. Cheng, C. A. Chang, and J. A. Yeh, "Varible focus dielectric liquid droplet lens," Opt. Express 14, 4101-4106 (2006).

13. H. Ren and S. T. Wu, "Tunable-focus liquid microlens array using dielectrophoretic effect," Opt. Express 15, 7140-7145 (2007).

14. B. Berge and J. Peseux, "Variable focal lens controlled by an external voltage: an application of electrowetting," Euro. Phys. J. E-Soft Mat. 3, 159-163 (2000).

15. N. Chronis, G. L. Liu, K. H. Jeong, and L. Lee, "Tunable liquid-filled microlens array integrated with microfluidic network," Opt. Express 11, 2370-2378 (2003).

16. J. Chen, W. Wang, J. Fang, and K. Varahramyan, "Variable-focusing microlens with microfluidic chip," J. Micromech. Microeng. 14, 675680 (2004).

17. A. Divetia, T.-H. Hsieh, J. Zhang, Z. Chen, M. Bachman, and G. P. $\mathrm{Li}$, "Dynamically focused optical coherence tomography for endoscopic applications," Appl. Phys. Lett. 86, 103902 (2005).

18. F. S. Tsai, S. H. Cho, W. Qiao, N. Kim, and Y. Lo, "Miniaturized unified imaging system using bio-inspired fluidic lens," Proc. SPIE 7061, 70610N (2008). 